\title{
Dynamic arm study: quantitative description of upper extremity function and activity of boys and men with duchenne muscular dystrophy
}

\author{
Mariska M. H. P. Janssen ${ }^{1 *}$, Jaap Harlaar ${ }^{2}$, Bart Koopman ${ }^{3}$ and Imelda J. M. de Groot ${ }^{1}$
}

\begin{abstract}
Background: Therapeutic management of upper extremity (UE) function of boys and men with Duchenne Muscular Dystrophy (DMD) requires sensitive and objective assessment. Therefore, we aimed to measure physiologic UE function of healthy subjects and DMD patients in different disease stages, and to evaluate the relation between these physiologic measures and functional UE scales.

Methods: Twenty-three DMD patients and twenty healthy controls (7-23 years) participated in this explorative case-control study. Maximal muscle torque, maximal and normalized surface electromyography (sEMG) amplitudes, muscle thickness, echogenicity and maximal passive and active joint angles were measured. At activity level, Brooke upper extremity rating scale and the Performance of Upper Limb (PUL) scale were used.

Results: Outcome measures related to proximal UE function could discriminate between disease stages. Increased normalized SEMG amplitudes were found in patients, even in early disease stages. Maximal active joint angles showed the strongest relation to Brooke scale $\left(R^{2}=0.88\right)$ and PUL scale $\left(R^{2}=0.85\right)$.

Conclusions: The decline of muscle functions precedes the decline in performance of UE activities, and therefore may play a role in early detection of UE limitations. Increased SEMG levels demonstrate that DMD patients use more of their muscle capacity compared to healthy subjects, to perform daily activities. This might result in increased fatigability. Active maximal joint angles are highly related to functional scales, so preserving the ability to use the full range of motion is important for the performance of daily activities. Close monitoring of active joint angles could therefore help in starting interventions that minimize functional UE decline in DMD patients timely.
\end{abstract}

Keywords: Duchenne muscular dystrophy, Upper limb, 3D motion analysis, Surface electromyography, Muscle ultrasound, Muscle torque

\section{Background}

Duchenne Muscular Dystrophy (DMD) is a $\mathrm{x}$-linked neuromuscular disorder with an incidence of 1:5,000 male newborns [1]. The disorder is characterized by a progressive loss of muscle strength, starting in the pelvic girdle, however, in later stages all muscles become affected. Boys with DMD become non-ambulant around

\footnotetext{
* Correspondence: Mariska.Janssen@radboudumc.nl

1Department of Rehabilitation, Radboud University Medical Center, Donders Centre for Neuroscience, Reinier Postlaan 4, 6525 GC Nijmegen, The Netherlands

Full list of author information is available at the end of the article
}

the age of 10 years when untreated, and around the age of 13 years when treated with corticosteroids [2]. Arm function is already affected at this age [3,4]. Although there is no curative treatment for DMD, life expectancy is rapidly increasing due to medical interventions [5, 6]. This means that boys and men with DMD have to live longer with their functional limitations and thus maintaining upper extremity (UE) function and measuring changes in UE function are increasingly important.

Loss of UE function can be delayed by several years by using corticosteroid treatment [7-10]. Physical exercise programs have also been found to be beneficial for 
retaining UE function [11-13]. However, in the long term, interventions that compensate for loss of UE function are still needed, for example arm supports, which reduce the effort that is needed to perform activities. To develop and evaluate such interventions, more insight in the upper extremity is needed. Insights on both International Classification of Functioning, Disability and Health (ICF) [14] function and structure level, and ICF activity level are necessary in order to unravel the mechanisms of UE decline.

The primary aim of this study is to give a quantitative description of UE functioning during a variety of meaningful UE task in boys and men with DMD in different stages of the disease, in comparison to their healthy peers. The secondary aim is to evaluate the relation between physiologic and structural UE functions and functional UE scales.

\section{Methods}

\section{Population}

The study population consisted of 23 boys and men with DMD and 20 healthy boys and men. DMD patients were included if they were older than 6 years, had a DNA established DMD diagnosis, and had a Brooke scale [15] of $1-5$, meaning that they were able to use their hands functionally. Patients were recruited through the Radboud University Medical Center (Radboudumc) outpatient clinic and by an advertisement on the website of the Dutch DMD patient organization ("Duchenne Parent Project"). Healthy subjects over 6 years, without UE mobility limitations, were included from schools in the neighborhood of the Radboudumc in the city of Nijmegen. This study was approved by the medical ethical committee Arnhem-Nijmegen, the Netherlands (Registration number 2012/135, NL nr.: 39126.091.12). Informed consent was obtained from all participants and from their parents when the subjects were under 18 years of age.

\section{Outcome measures \\ Participant characteristics}

The following participant characteristics were collected based on self-reports: age, arm preference, weight, height, year of diagnosis, wheelchair confinement and, if applicable the age of wheelchair confinement, and the occurrence of scoliosis.

\section{Functional UE scales}

Functional UE scales used in this study were: "Brooke upper extremity rating scale [15]" and the "Performance of Upper Limb (PUL) scale [16]". These functional scales measured participants' activity level. PUL items were performed once. Based on the score of the entry item, some subjects only performed a specific subset of the PUL. Sum scores of the 3 dimensions (high level shoulder, mid level elbow, distal wrist and hand) and the total sum score were calculated.

\section{Muscle torques and surface electromyography}

Muscle torques and surface electromyography (sEMG) signals were recorded of 7 different upper extremity muscles (Trapezius (descending part), Biceps Brachii (long head), Triceps Brachii (long head), Deltoid (lateral part), Pectoralis Major (clavicular head), wrist flexors and wrist extensors). Muscle torques were measured using a static frame myometer, consisting of a KAP-E Force Transducer, measurement range $0.2-2000 \mathrm{~N}$ (Angewandte System Technik, Dresden, Germany), and a height and position adjustable frame (designed and custom made by mechanical engineers from the VU medical centre, Amsterdam, the Netherlands). Wireless sEMG signals (Zerowire EMG, Aurion, Italy) were recorded with a sample frequency of $1000 \mathrm{~Hz}$. Disk-shaped Ag-AgCL ARBO ECG electrodes (Tyco Healthcare, Neustadt, Germany) were placed at an inter electrode distance of $24 \mathrm{~mm}$. Testing and electrode positions were based on literature $[17,18]$. To make the measurement protocol more suitable for DMD patients, as they were often in a wheelchair or had joint contractures, we slightly adapted some of the testing positions. sEMG data were filtered using a $4^{\text {th }}$ order band pass filter between 20 and $450 \mathrm{~Hz}$, where after the signal was rectified and low pass filtered $(3 \mathrm{~Hz})$ to obtain the linear envelope $[19,20]$. Torque data were filtered using a $3 \mathrm{~Hz}$ low pass filter of the $4^{\text {th }}$ order.

All subjects performed two maximal voluntary isometric contractions (MVICs) to determine the maximal muscle torque and corresponding sEMG amplitude. If the examiner was not confident that a maximal effort was made, the measurement was repeated. The maximal value out of the two correct attempts was used for further data analysis. Normalized sEMG amplitudes were calculated for the performance of single joint movements and PUL items. Normalized sEMG amplitude was defined as the maximum sEMG amplitude that was reached during a movement as a percentage of the maximal amplitude of the same muscle during MVIC.

Data was processed with custom-written Matlab (Matlab ${ }^{\circ}$ version R2014b, Mathworks, Natick, USA) routines.

\section{Quantitative muscle ultrasound}

Ultrasounds images of 6 upper extremity muscles (Trapezius, Biceps Brachii, Triceps, Deltoid, wrist flexors and wrist extensors) were recorded using a Z.One PRO Ultrasound System (Zonare Medical Systems, Mountain View, California, USA), with a L10-5 transducer. Three ultrasound recordings were made, at a depth of $4 \mathrm{~cm}$, to calculate echogenicity (greyscale) and one recording, with no predefined depth, was made to determine the muscle thickness. Echogenicity is the extent to which a 
structure reflects ultrasound of a surface with high echogenicity indicating that more ultrasound is reflected, for example when high levels of fatty and connective tissue are present in a muscle. Ultrasound images were analyzed with computer-assisted greyscale histogram analysis, using custom software developed at Radboudumc (QUMIA). Echogenicity was determined by calculating the grayscale in the upper $1 / 3^{\text {rd }}$ of the region of interest (the region that included as much muscle mass as possible without bone and fascia) in each muscle [21]. The average echogenicity out of 3 measurements was used for further analysis. Muscle thickness was determined by calculating the distance between two electronic calipers at standardized positions. Thickness of the Trapezius was measured between the deep and superficial fascia of the upper part of the Trapezius muscle. Thickness of the Deltoid, Biceps (combined with Brachialis) and Triceps muscles were measured between the humerus and the superficial fascia. Forearm flexor (Flexor Carpi Radialis) thickness was measured between a horizontal reference line at the height of the radius and the superficial fascia. Forearm extensors thickness was measured between the middle end of the radius and the superficial fascia.

Ultrasound results were compared to muscle specific reference values and expressed as Z-scores (representing the number of standard deviations from the mean) [22]. Reference values for calculation of the Z-scores were obtained from 60 healthy subjects using the same measurement protocol and ultrasound device (manuscript in preparation). Echogenicity and muscle thickness were corrected for age, weight and height if necessary using the method described by Scholten et al. [23].

\section{Three dimensional motion analysis}

Three dimensional motion analysis, using the kinematic model of Jaspers et al. [24] (Fig. 1), was performed with an 8 camera VICON motion analysis system (Oxford Metrics, Oxford, UK). After marker placement and anatomical landmark identification, maximal passive joint angles were determined for: 'shoulder abduction', 'elbow flexion and extension', 'pro- and supination of the lower arm, 'wrist flexion and extension' and 'wrist ulnar and radial deviation'. Maximal active joint angles were determined for the same movements and also for 'shoulder flexion' and 'shoulder adduction (in the horizontal plane)' (Fig. 2). Some subjects did not perform all single joint movements as they were unable to perform the movements. All passive and active movements were performed 3 times at a controlled movement velocity.

Joint-kinematics were calculated using BodyMech (http://www.bodymech.nl) and additional custom-written Matlab routines. Kinematic data were filtered using a $4^{\text {th }}$ order low pass filter of $20 \mathrm{~Hz}$. Per movement, the minimal and maximal joint angles were determined. The average

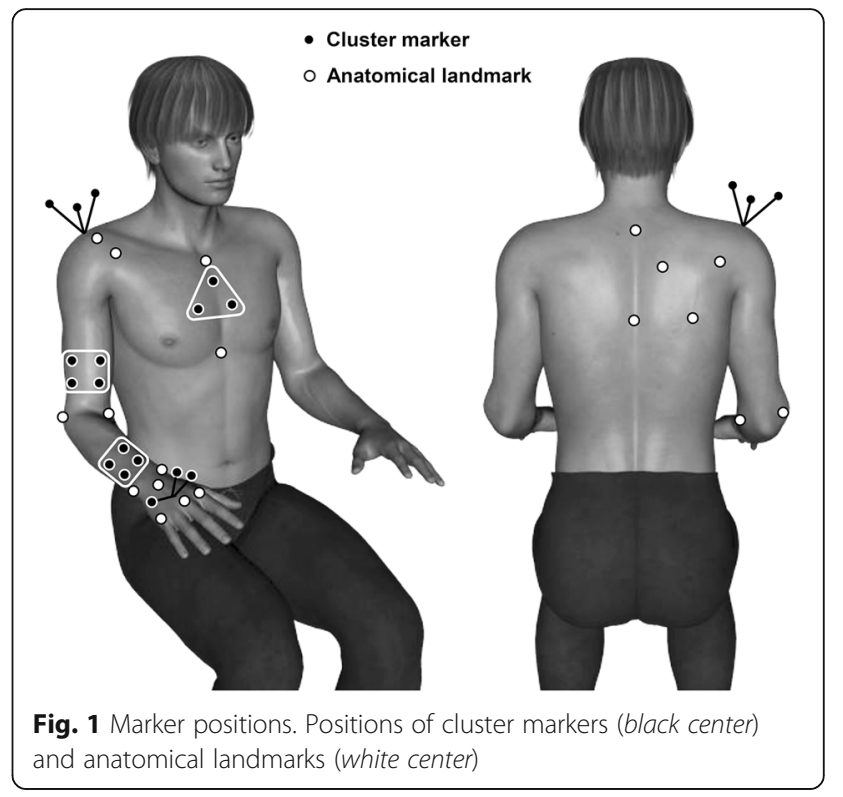

maximal joint angle over three measurements was used for further data analysis.

\section{Statistical analysis}

Median values and ranges were used to describe the continuous participant characteristics and percentages were used to describe categorical participant characteristics. Wilcoxon rank sum tests were used to compare outcome measure sum scores between healthy subjects and DMD patients. Kruskal-Wallis tests were used to test for differences between DMD patients in different Brooke scales. To gain insight in the relation between functional UE scales (Brooke and PUL scale) and physiologic UE function (muscle torque, sEMG, echogenicity, muscle thickness, passive and active joint angles) we calculated the coefficient of determination $\left(R^{2}\right)$ between the sum scores, or average scores for echogenicity and muscle thickness, of these outcome measures. The sum scores were calculated by adding the results of all values within one outcome measure. If one or more values were missing, the sum score was also reported as missing. If values were missing because patients were physically unable to perform the activity a score of 0 was used for the calculation of the sum scores. SPSS Statistics Version 20 (IBM, Somers, USA) was used for statistical analysis.

\section{Results}

The median age of healthy subjects was 14.0 (range 7.4-23.4) years and the median age of DMD patients was 14.9 (range 8.1-21.7) years (Table 1). About $90 \%$ of the participants was right handed. The median age at diagnosis was 3.75 years (range $0-7$ years) and $74 \%$ of the patients was non-ambulant. Thirteen percent of the patients had a mild scoliosis, and $22 \%$ had a severe 

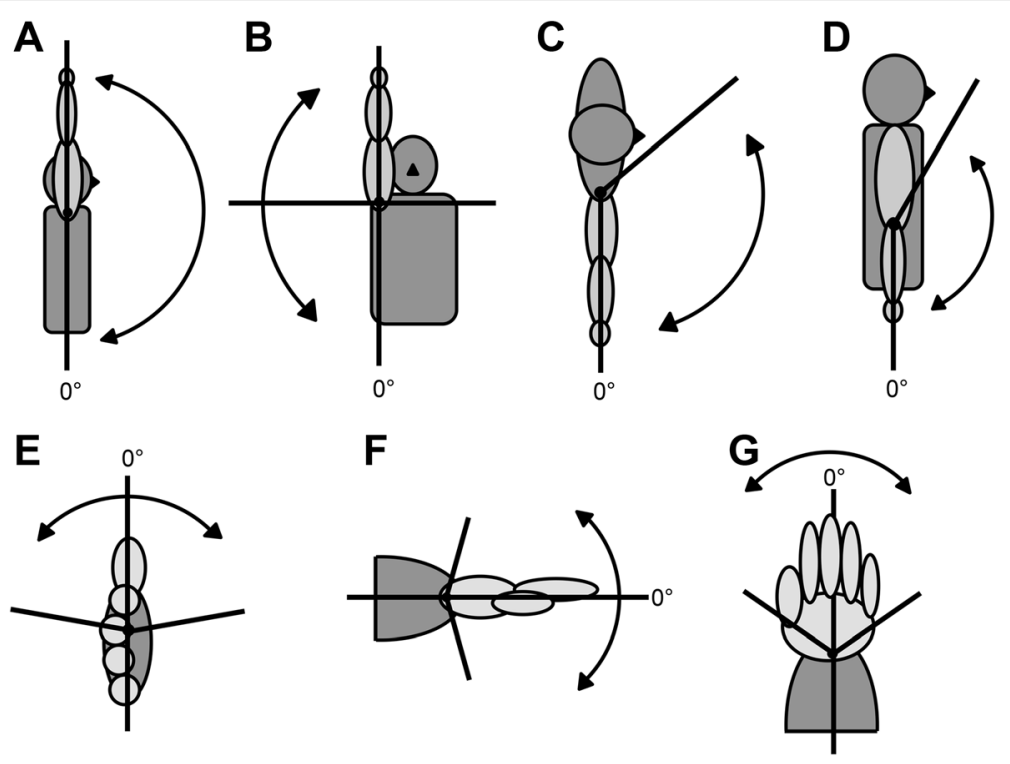

Fig. 2 Single joint movements. a shoulder flexion, (b) shoulder abduction, (c) shoulder adduction (in the horizontal plane), (d) elbow flexion and extension, (e) forearm pronation and supination, (f) wrist flexion and extension and (g) wrist ulnar and radial deviation

scoliosis, of which $40 \%$ was surgically corrected. Corticosteroids were used by $74 \%$ of the patients, while $13 \%$ stopped using and 13\% never used corticosteroids. Of the corticosteroid users, $12 \%$ used Deflazacort on a daily basis and $88 \%$ uses Prednisone/Prednisolone on a 10-days-on/ 10-days-off basis. Dosages vary between 4 and $45 \mathrm{mg}$.
Statistically significant differences between healthy subjects and DMD patients were seen in all outcome measures except muscle thickness, as all the Z-values for muscle thickness were between -2 and 2 (Table 2). In addition, differences between patients in different Brooke scales were present in most proximal muscles

Table 1 Participant characteristics

\begin{tabular}{|c|c|c|c|c|c|c|}
\hline Variable & Healthy & DMD Brooke 1 & DMD Brooke 2 & DMD Brooke 3 & DMD Brooke 4 & DMD Brooke 5 \\
\hline $\bar{N}$ & 20 & 5 & 8 & 4 & 3 & 3 \\
\hline Age (median, range) & $14.0(7.4-23.4)$ & $11.1(8.0-16.0)$ & $12.4(8.7-15.8)$ & $15.8(12.6-16.9)$ & $17.1(17.0-18.4)$ & $18.2(17.8-21.7)$ \\
\hline BMl & $19.1(15.7-24.4)$ & $21.9(17.7-26.4)$ & $20.7(18.7-30.8)$ & $23.0(16.5-26.0)$ & $17.6(10.0-21.4)$ & $22.5(19.4-24.6)$ \\
\hline \multicolumn{7}{|l|}{ Hand preference } \\
\hline Right handed (\%) & 90 & 100 & 87.5 & 75 & 100 & 100 \\
\hline Left handed (\%) & 10 & 0 & 12.5 & 25 & 0 & 0 \\
\hline Age of diagnosis (median, range) & - & $2.0(0.0-5.0)$ & $4.0(2.5-7.0)$ & $4.0(2.5-6.0)$ & $1.5(0.0-6.0)$ & $1.0(0.0-6.0)$ \\
\hline Percentage wheelchair confined (\%) & - & 0 & 87.5 & 100 & 100 & 100 \\
\hline Age wheelchair confined (median, range) & - & - & $10(7-13)$ & $10(8-10)$ & $11(10-11)$ & $9(8-10)$ \\
\hline \multicolumn{7}{|l|}{ Scoliosis } \\
\hline No (\%) & - & 75 & 87.5 & 75 & 33 & 0 \\
\hline Mild (\%) & - & 25 & 12.5 & 0 & 0 & 33 \\
\hline Severe (\%) & - & & & 25 & 67 & 67 \\
\hline Scoliosis correction (\%) & - & 0 & 0 & 0 & 33 & 67 \\
\hline \multicolumn{7}{|l|}{ Corticosteroid use } \\
\hline No (\%) & - & 0 & 12.5 & 25 & 33 & 0 \\
\hline Not anymore (\%) & - & 0 & 0 & 25 & 0 & 67 \\
\hline Yes (\%) & - & 100 & 87.5 & 50 & 67 & 33 \\
\hline
\end{tabular}




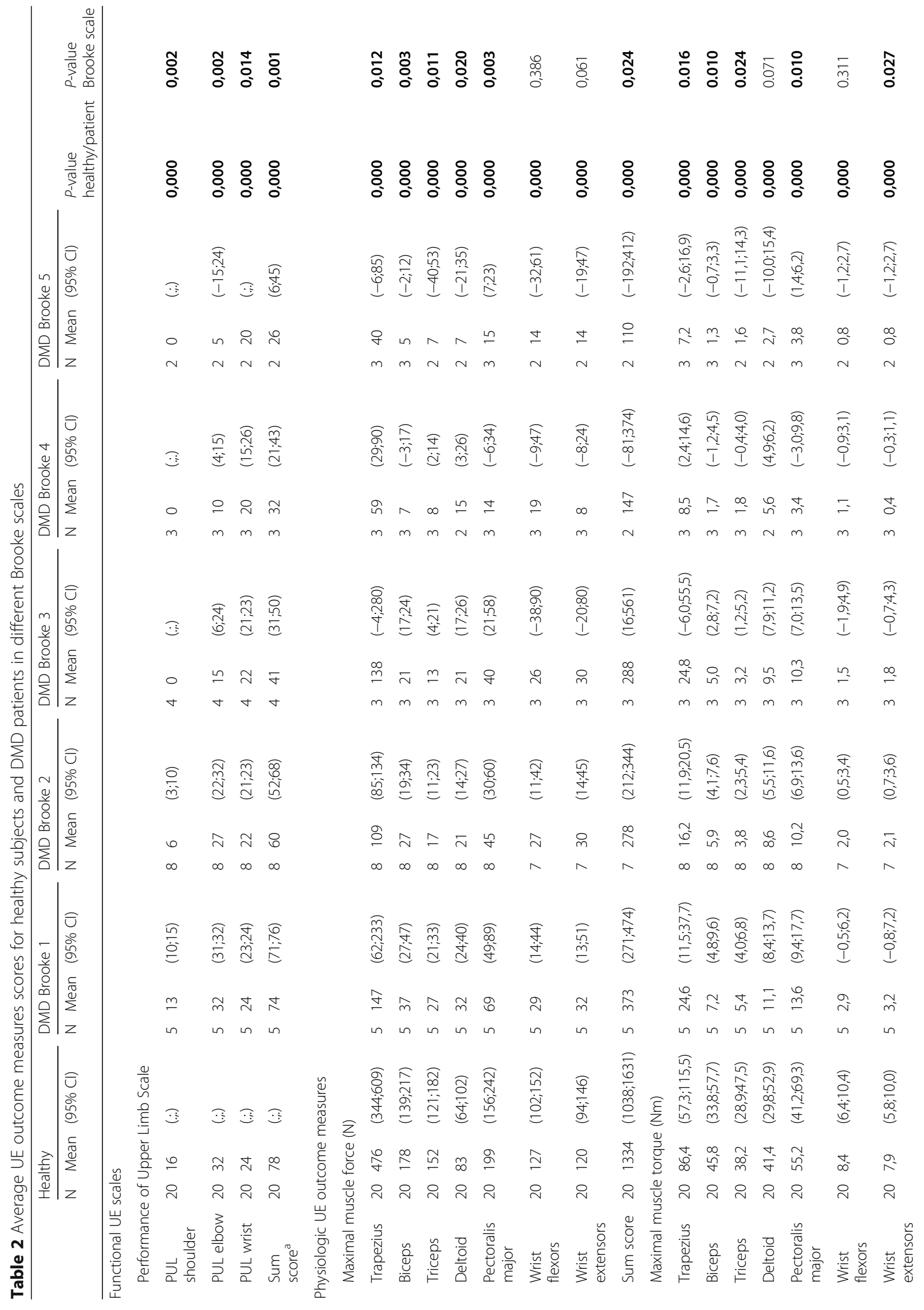




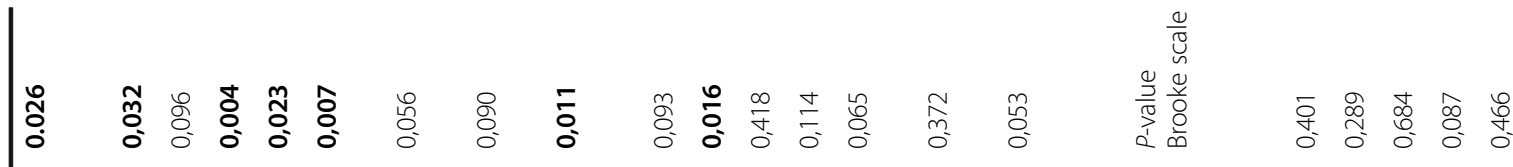

章

ลิ

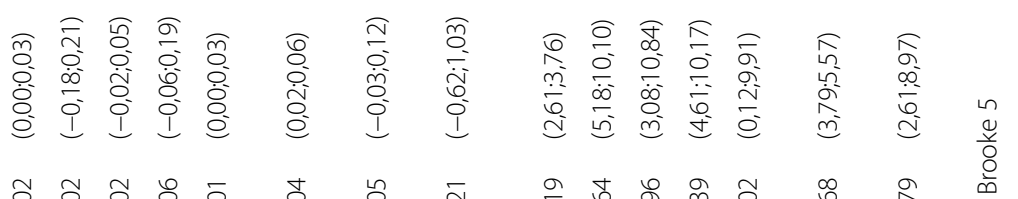

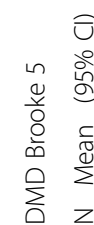

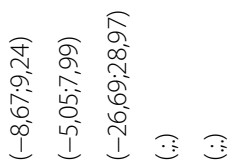

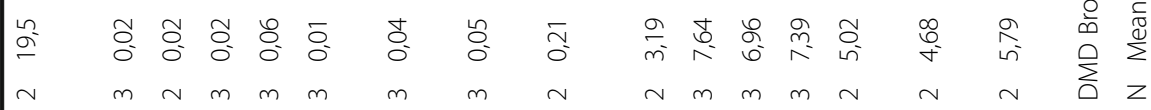

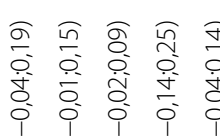

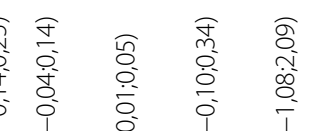

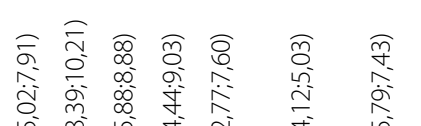

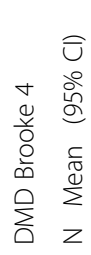

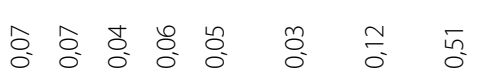

f્t

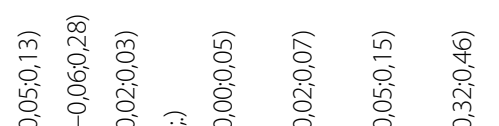

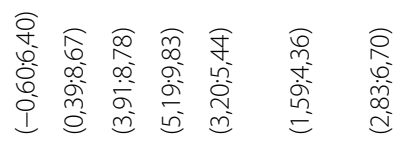

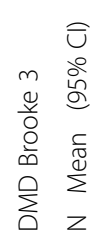

尺े f

$\sim m \sim 0-$

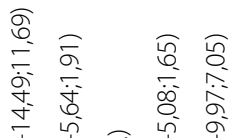

I i I i i

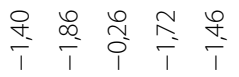

$\sim m-\sim \sim$

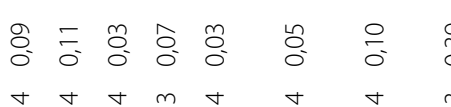

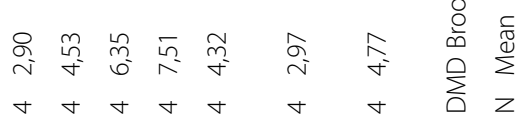

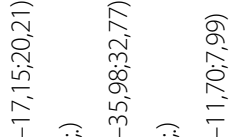

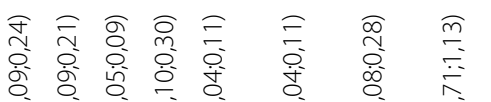

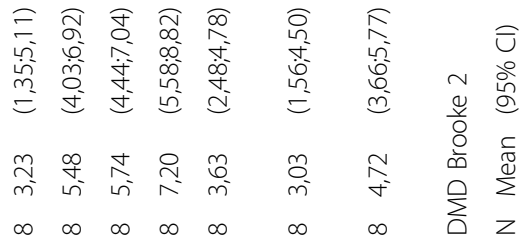

\section{๑}

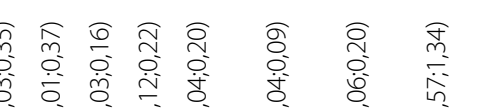

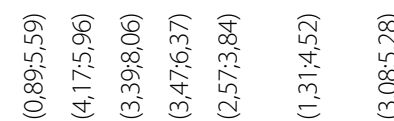

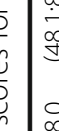

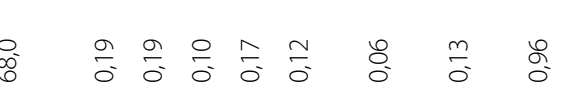

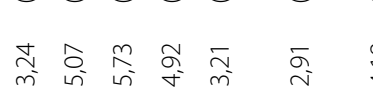

un in un un th

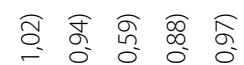

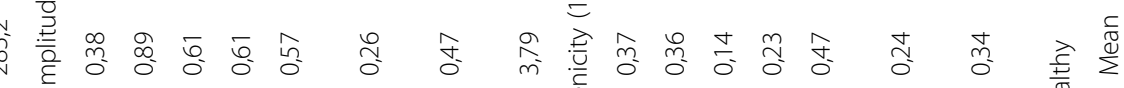

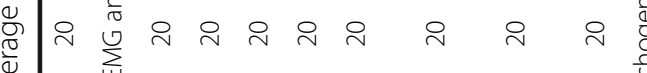




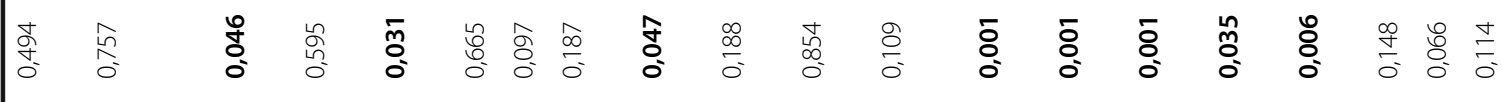

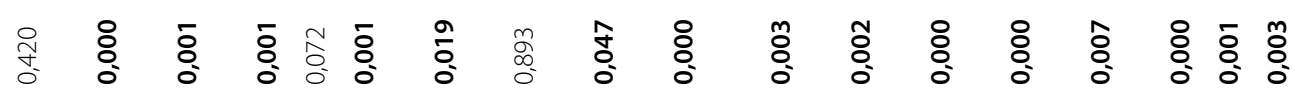

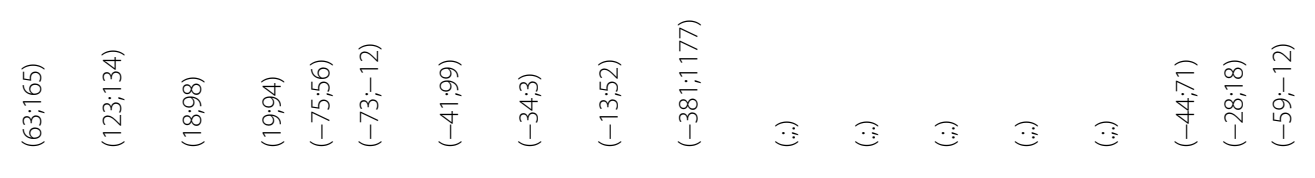

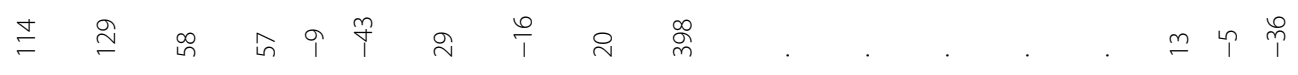

จิ

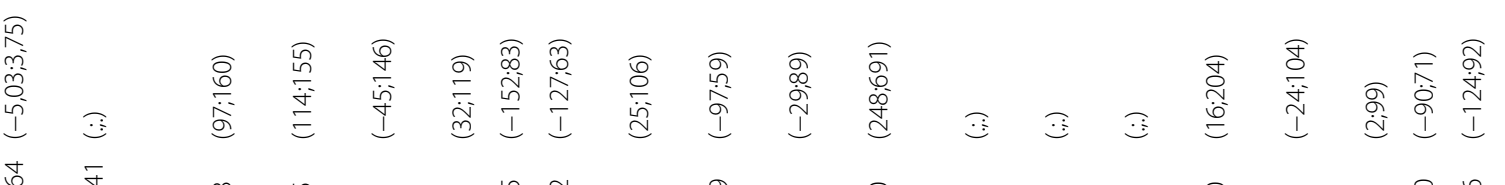

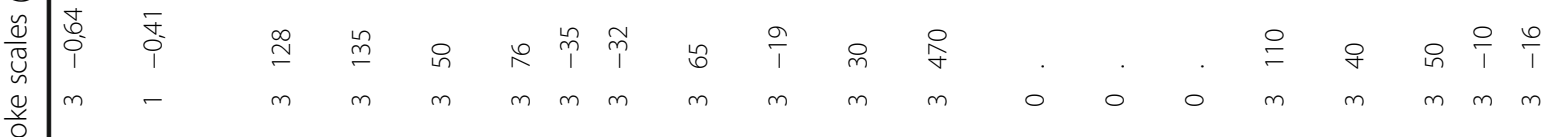

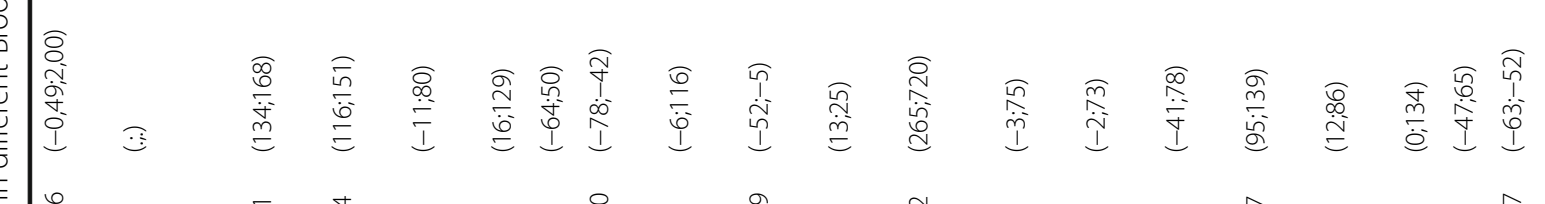

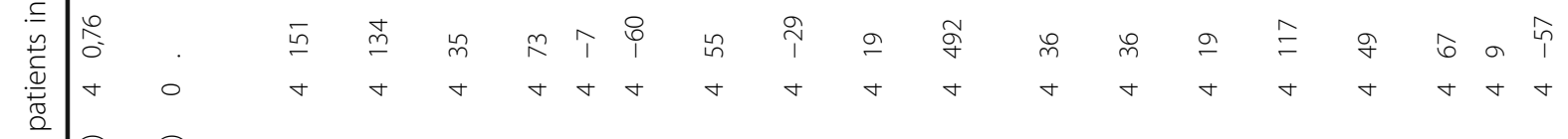

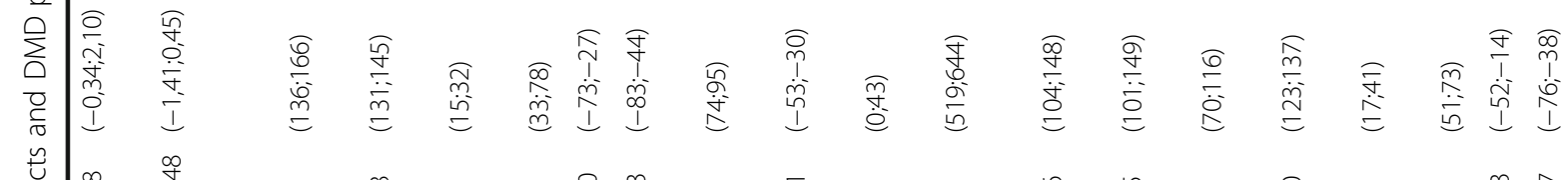

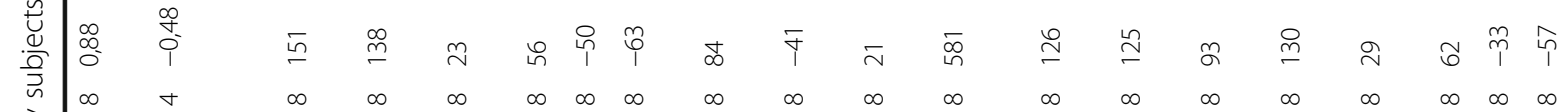

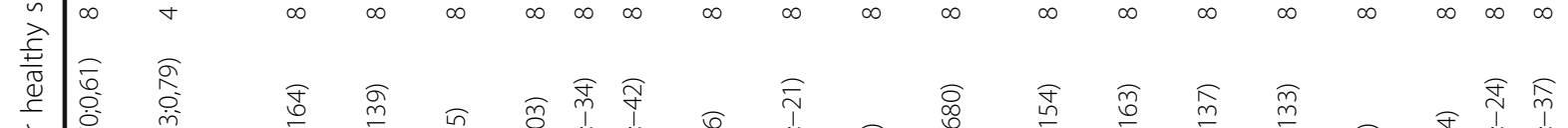

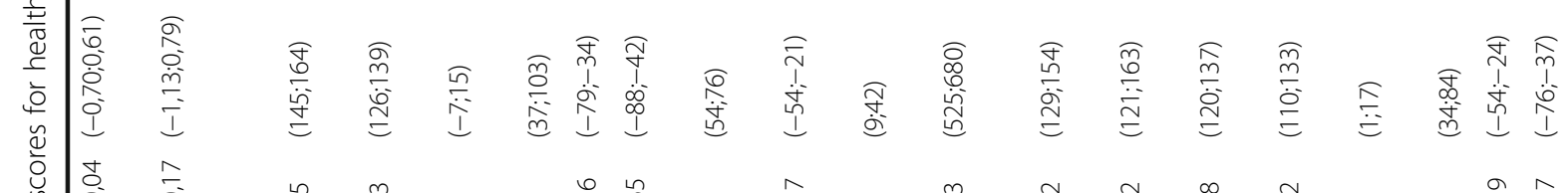

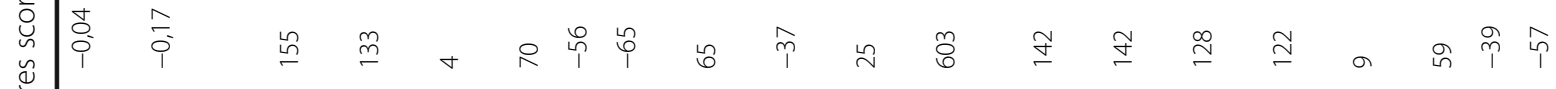

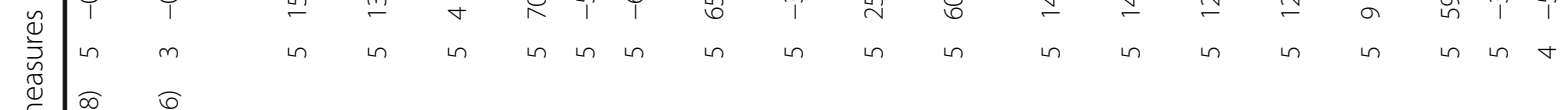

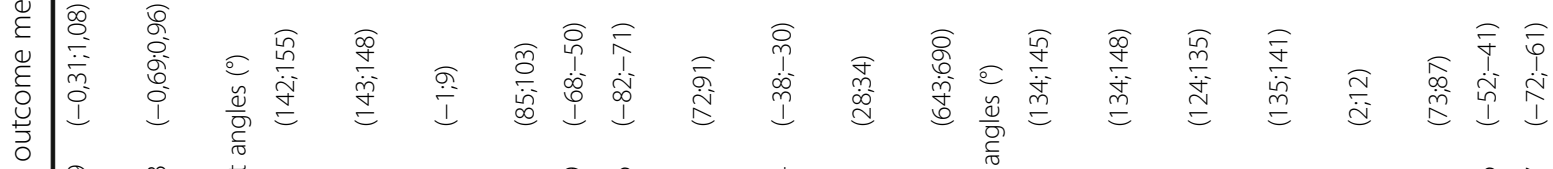

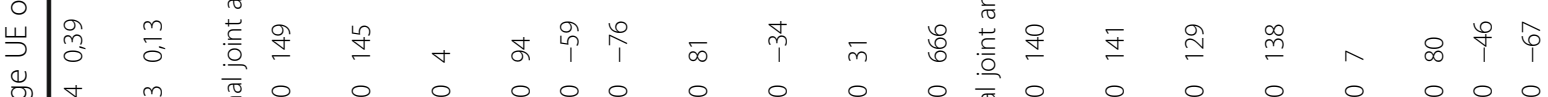

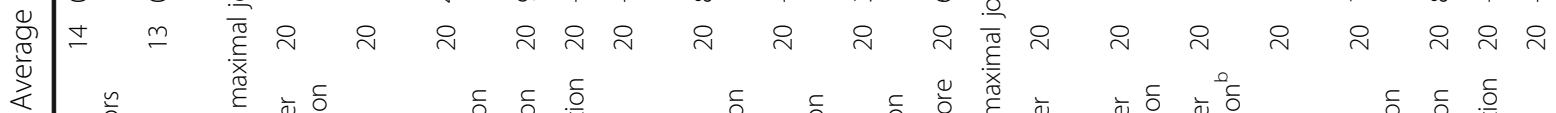

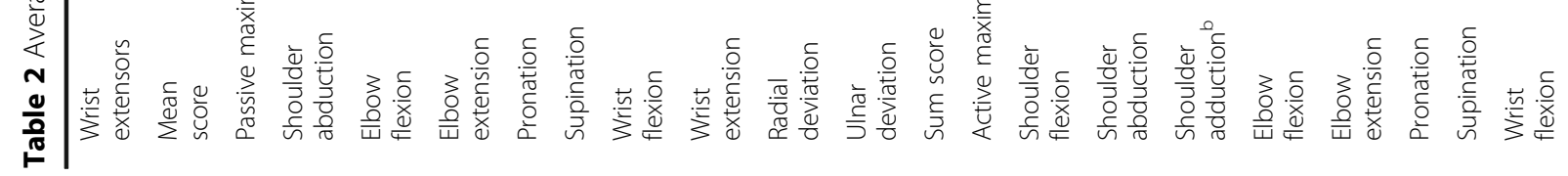




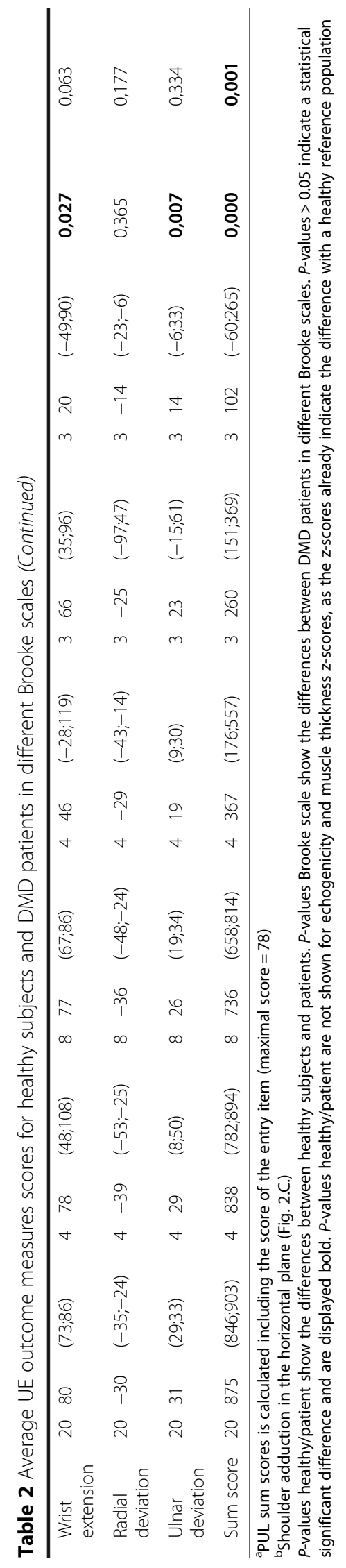


and movements requiring proximal muscles. PUL scores in all domains differed between DMD patients in different Brooke scales.

Normalized sEMG amplitudes of DMD patients and healthy controls differed significantly for all movements and muscles, except for Trapezius activation during shoulder abduction (Fig. 3). Maximal active joint angle sum score shows the strongest correlations with Brooke scale (Fig. 4) and PUL score (Fig. 5) $\left(R^{2}\right.$ of 0.88 and 0.85 respectively), followed by maximal muscle torque and maximal sEMG amplitude sum scores $\left(R^{2}>0.5\right)$. Echogenicity and passive maximal joint angle sum scores explain about $30 \%$ of the variance of Brooke scale and PUL score.

In healthy subjects, a strong relation with age was present for maximal muscle torque sum score and mean muscle thickness z-score (Fig. $6, R^{2}$ of 0.79 and 0.86 respectively). For DMD patients the strongest correlations with age were found for maximal active joint angle sum score and Brooke scale ( $R^{2}$ of 0.64 and 0.63 respectively).

\section{Discussion}

Our study provides new insights in the muscles and movements that are affected most in DMD patients, and how this relates to functional UE scales. This is vital information for clinical decision making, but can also be used in the development of new outcome measures in clinical trials.

Currently, functional scales such as the Performance of Upper Limb (PUL) scale and the Motor Function Measure (MFM) are used as the gold standard for quantifying UE limitations in DMD. These measures, however, are not able to cover the entire spectrum of DMD patients, as they have floor and ceiling effects [16]. Furthermore, they do not give insight in the underlying working mechanisms of the UE. Daily activities require sufficient strength of multiple muscle groups and motion in multiple joints. Therefore, functional scales give insight in problems that result from a combination of many different physiologic aspects of UE function.

Our study shows that muscle functions (i.e. maximal muscle torque, maximal sEMG amplitude and echogenicity z-scores) of DMD patients already deviate from healthy subjects in an early disease stage (i.e. Brooke 1). A similar reduction of muscle force/torque in young DMD patients has been reported in previously [3, 25-27]. Echogenicity z-scores of all muscles are above two thus differ significantly from the healthy reference population. This finding indicates that muscles are infiltrated with fatty and connective tissue, which is in line with the results of other studies [21, 28, 29]. Consequently, these outcome measures are of great importance for early detection of UE
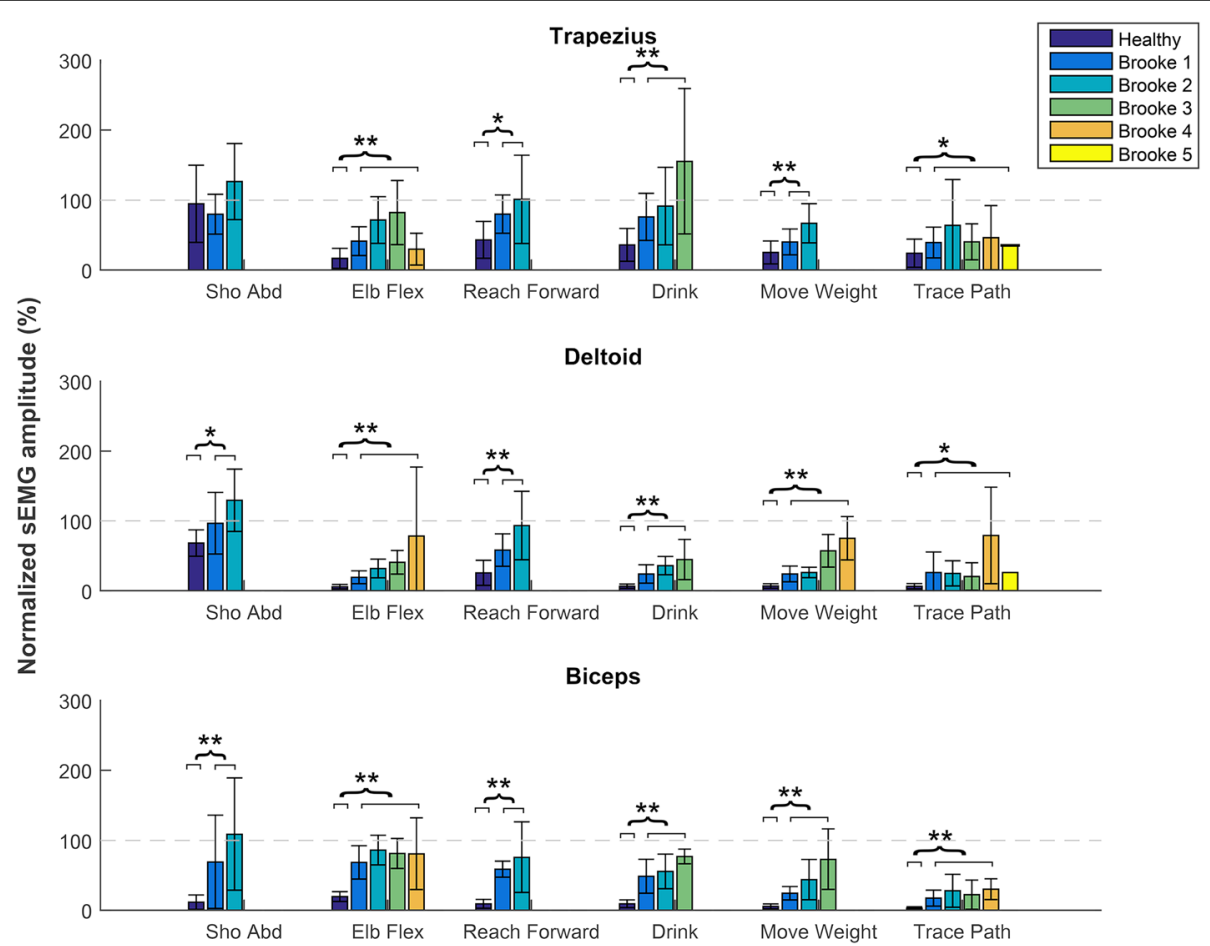

Fig. 3 Normalized sEMG amplitudes. Normalized sEMG amplitudes of the Trapezius, Deltoid and Biceps Brachii muscles for 6 different upper extremity movements shown for healthy subject and DMD patients in different Brooke scales. Sho Abd= shoulder abduction; Elb Flex = elbow flexion; Reach Forward = reaching forward at shoulder level without weight (PUL item D); Drink = drinking from a full cup (200 g) (PUL item F); Move Weight = moving a $100 \mathrm{~g}$ weight (PUL item H); Trace Path = tracing a path (PUL item O) 


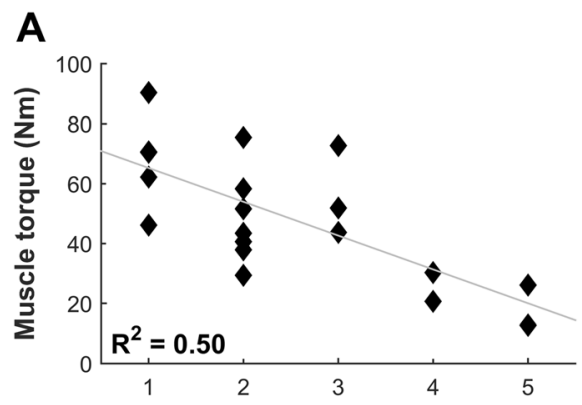

C

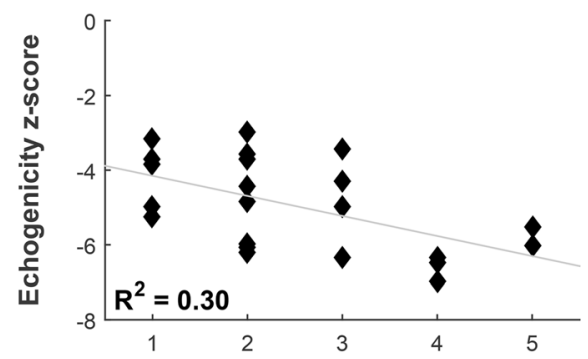

$\mathbf{E}$

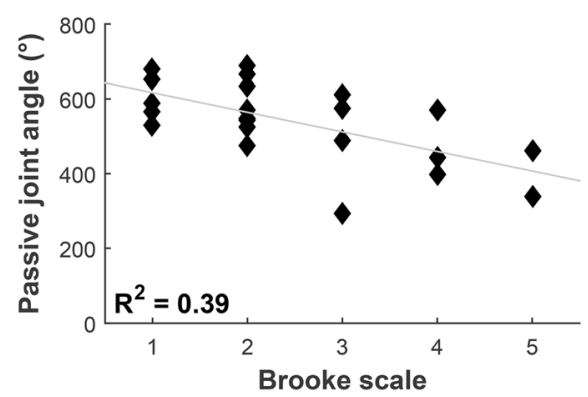

B

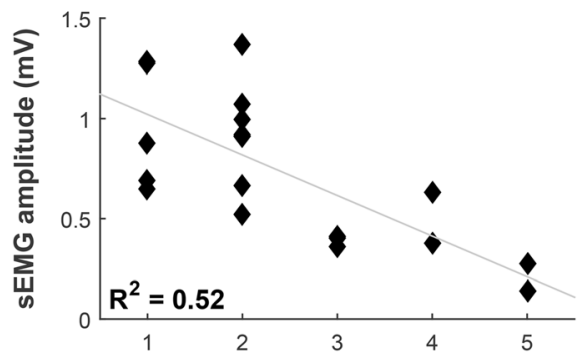

D

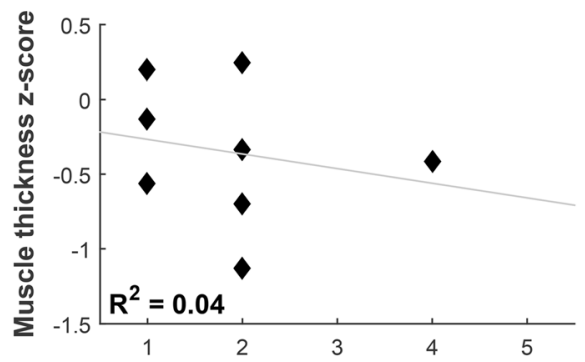

$\mathbf{F}$

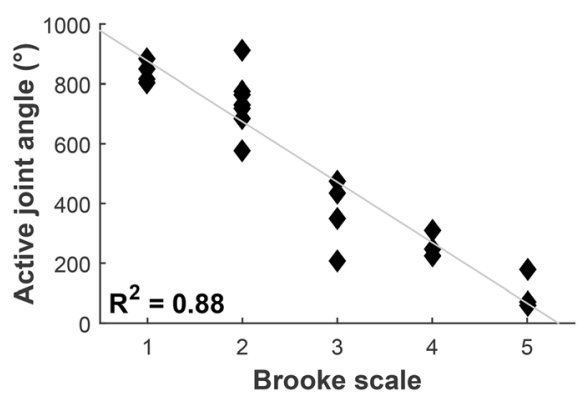

Fig. 4 Correlations with Brooke scale. Correlations of DMD patients between Brooke score and (a) maximal muscle torque sum score; b maximal sEMG amplitude (MVIC) sum score; $\mathbf{c}$ mean inverse z-score of echogenicity (inverse z-scores were used so that lower scores indicate worse UE function); $\mathbf{d}$ mean $z$-score of muscle thickness; e maximal passive joint angle sum score; $\mathbf{f}$ maximal active joint angle sum score

impairments, as activity scales cannot be used in the earliest disease stage due to ceiling effects. Early detection is important to start interventions early, for example physical exercise training, which is proven to be effective in delaying functional deterioration [11-13]. The current study shows that mainly proximal muscles and movements requiring proximal muscle activation are sensitive to detect differences of UE function and activity. Maximal muscle torques and maximal sEMG amplitudes of proximal muscles can also detect differences in the later disease stages (Brooke 4 and 5), even though the muscles cannot initiate movements anymore. This could be important for evaluating the effects of arm supports, or other interventions aimed at late stage DMD patients.

To identify which limitations are primarily responsible for the inability to perform activities, and how this relates to weakness in specific muscles, insight in single joint movements is important. Single joint movements consist of movements over one joint, which often can be related to the activation of one primary muscle. In clinical practice, the measurement of maximal active single joint angles can give more insight in the mechanism responsible for activity limitations. This statement is supported by the very strong relation we found between maximal active joint angles and PUL score $\left(R^{2}=0.85\right)$.

Our results show that when the maximal Deltoid torque drops below approximately $10 \mathrm{Nm}$, DMD patients start to have difficulties lifting their arms. A maximal Biceps torque below approximately $5 \mathrm{Nm}$ is related to restrictions in elbow motion. It is likely these are the minimum torques required to move the upper/lower arm against gravity, and could help to identify the suitable time to start using an arm support. Hence, regular assessment of deltoid and biceps torques may help clinicians plan interventions, anticipating functional decline.

We found that active and passive joint angles decline almost simultaneously. Therefore, we hypothesize that when a patient loses the ability to move a joint actively, 

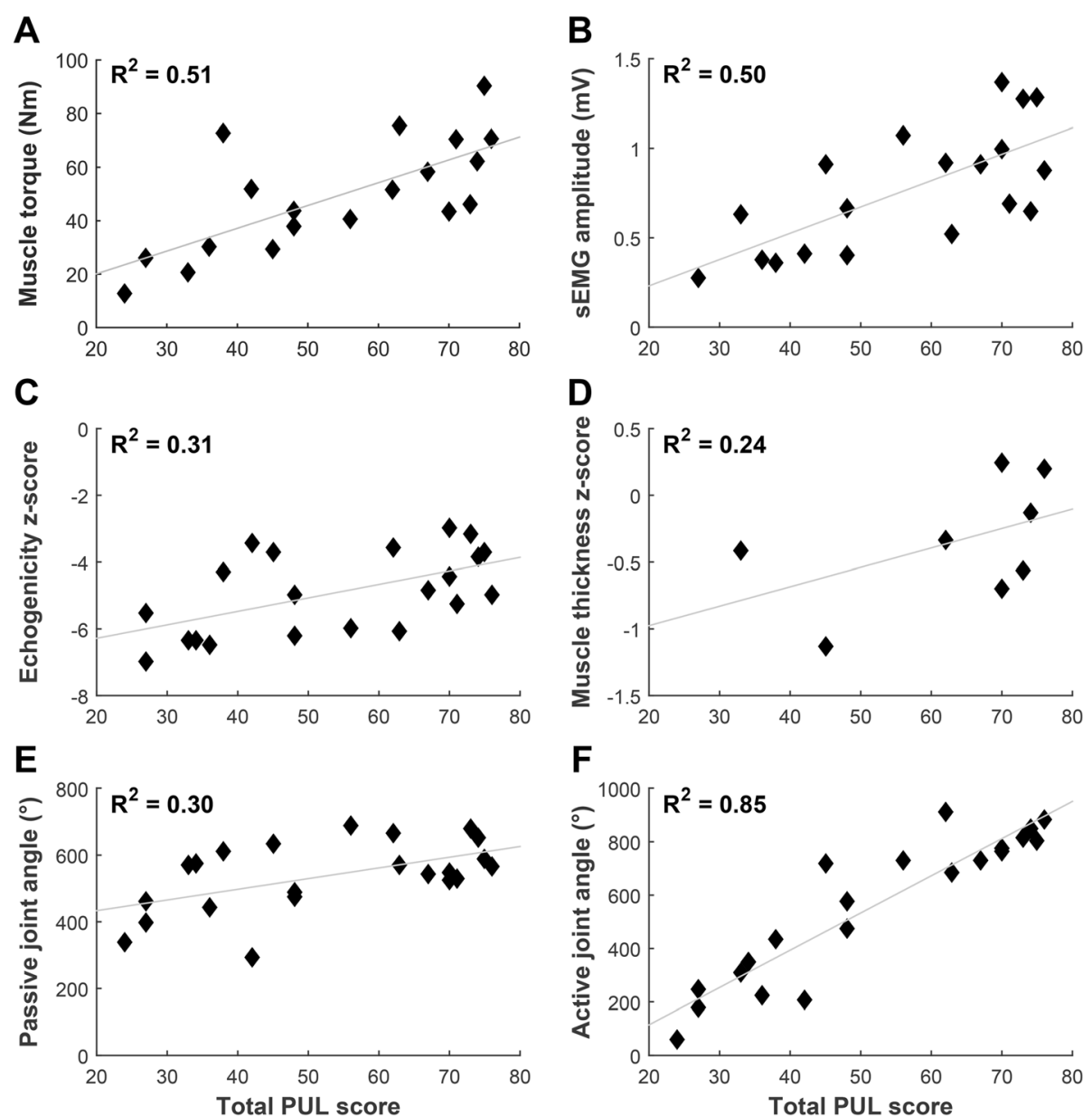

Fig. 5 Correlations with PUL score. Correlations of DMD patients between total PUL score and (a) maximal muscle torque sum score; $\mathbf{b}$ maximal sEMG amplitude (MVIC) sum score; c mean inverse z-score of echogenicity (inverse z-scores were used so that lower scores indicate worse UE function); $\mathbf{d}$ mean z-score of muscle thickness; e maximal passive joint angle sum score; $\mathbf{f}$ maximal active joint angle sum score

the joint will be statically positioned for longer periods, which leads to contractures soon thereafter. This hypothesis is in line with the findings of McDonald et al. who showed the occurence of elbow flexion contractures appears to be related to static positions of the limb after wheelchair confinement [30]. Hence, we recommend to start interventions, such as stretching exercises, as soon as active joint angles start to decrease. In addition, we recommend stimulation of (supported) movement to limit static positioning and thereby prevent contracture formation [31].

A recent study has indicated that fatigue was strongly associated with health-related quality of life and that there should be a greater clinical focus on the reduction of fatigue [32]. In this study, we measured maximal sEMG amplitudes, which is a measure for the maximal muscle capacity. Normalized sEMG amplitudes show the percentage of this maximal muscle capacity needed to perform activities. When normalized sEMG amplitudes are high, a larger percentage of the muscle capacity is used, which leads to faster occurrence of fatigue [33]. Our results show that DMD patients use a larger percentage of their muscle capacity to perform movements and activities compared to healthy subjects, even in an early stage of the disease, and therefore might experience earlier and more fatigue. This increase in the percentage of muscle capacity is not only seen in prime movers, but also in secondary movers indicating the use of compensatory muscles to overcome loss of muscle strength. Future studies should try to determine normalized sEMG amplitudes and normalized sEMG median frequency during a fatigue protocol, in order to gain more insight in muscle fatigue of DMD patients compared to healthy controls.

Although most of our results are in line with existing literature, we also found some differences. The passive forearm supination angle of DMD patients in this study did not differ significantly $(p=0.072)$ from healthy 

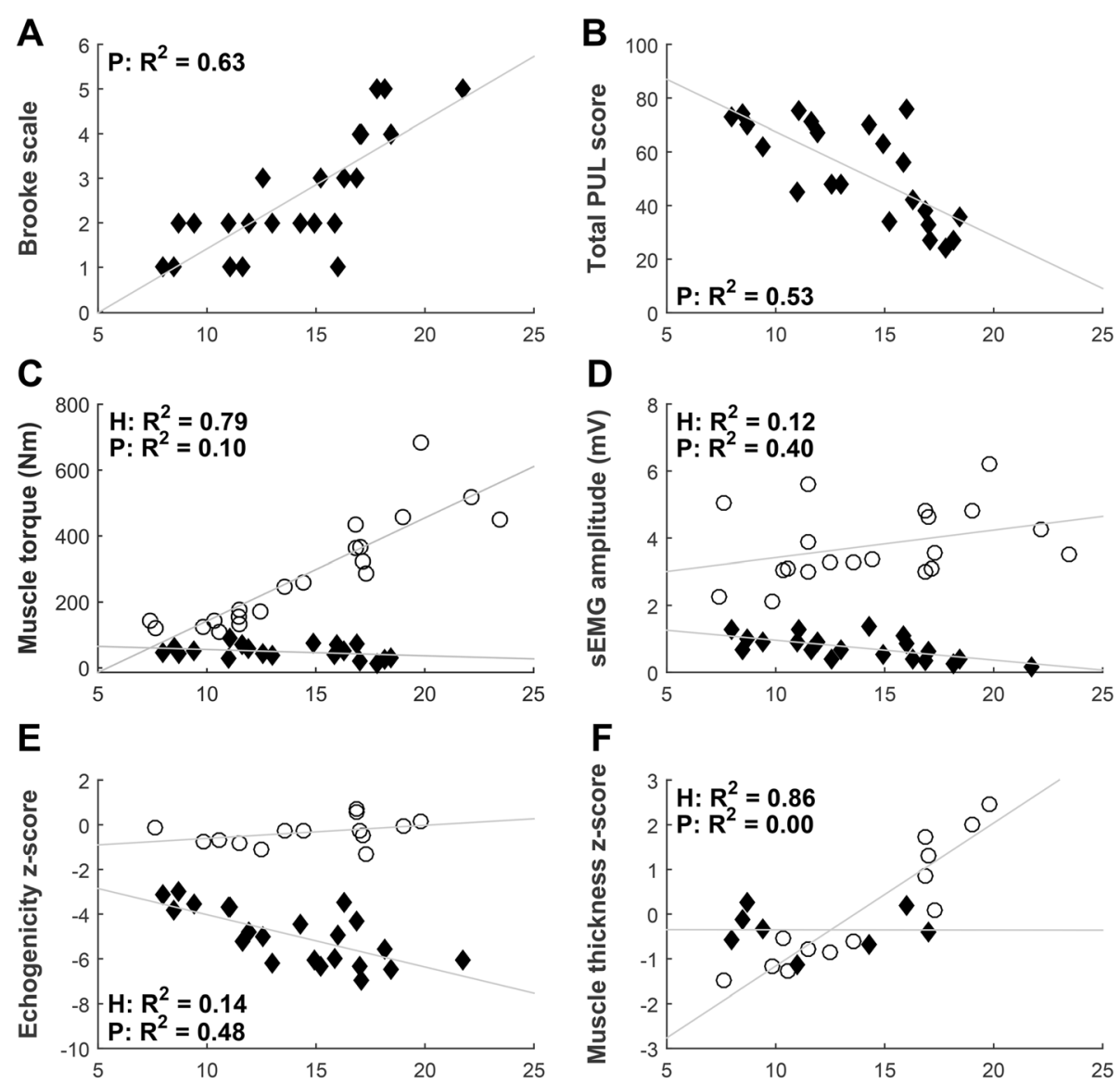

G
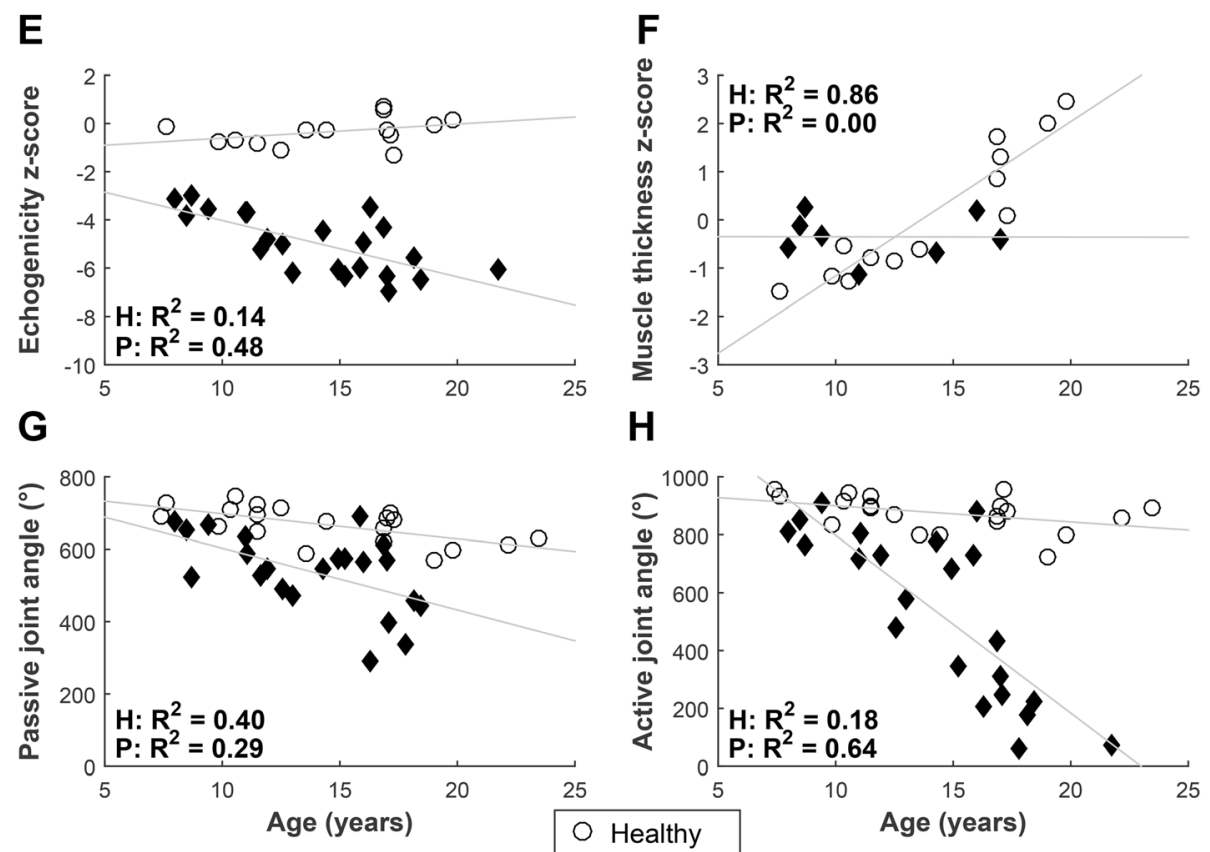

H

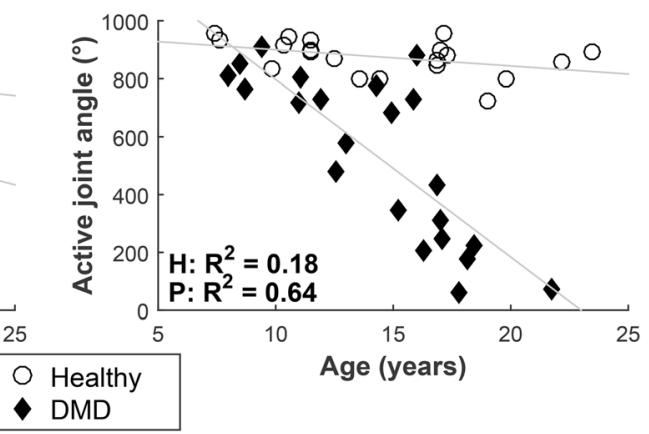

Fig. 6 Correlations with age. Correlations of DMD patients and healthy subject between age and (a) Brooke scale; $\mathbf{b}$ total PUL score; $\mathbf{c}$ maximal muscle torque sum score; $\mathbf{d}$ maximal sEMG amplitude (MVIC) sum score; e mean inverse z-score of echogenicity (inverse z-scores were used so that lower scores indicate worse UE function); $\mathbf{f}$ mean $\mathbf{z}$-score of muscle thickness; $\mathbf{g}$ maximal passive joint angle sum score; $\mathbf{h}$ maximal active joint angle sum score

subjects, as opposed to findings from Bartels et al. [27]. However, we found that the average forearm supination angles were reduced in patients from Brooke 3 onward, which is in line with the results of Bartels et al. who reported that $83 \%$ of the adult men with DMD had loss of supination [27]. As far as we are aware, the differences we found between healthy subjects and DMD patients for passive elbow flexion, forearm pronation, wrist flexion and ulnar deviation have not been reported before. Although the differences between healthy subjects and DMD patients are small, they could be of clinical relevance for the performance of daily UE activities [34-36].

Muscle ultrasounds are able to make distinction between different stages of DMD [21, 28] We, however, found that echogenicity is less strongly related to disease stage compared to maximal muscle torque and maximal 
sEMG amplitude. We expect that echogenicity, which is a measure for muscle degeneration, is less discriminative in the explored muscles because the ultrasound images are heavily affected by attenuation. This is especially true for the later disease stages, as an increased amount of fat and connective tissue in the muscles prevents the ultrasound from penetrating deeper layers of the muscle, which results in a darker picture and therefore lowers Zscores. For the same reason muscle thickness could not be measured accurately in older patients.

\section{Study limitations}

A limitation of this study is the relatively small number of patients in each group, especially in the latest disease stages. For this reason, post hoc comparisons between different disease stages were not performed. Furthermore, stratification of possible confounders, such as corticosteroid use and scoliosis, was not possible due to the small sample size. In addition, as this study is cross-sectional, we were unable to determine longitudinal changes of UE function. Therefore we recommend future UE studies to monitor changes of physiologic UE function over time in a cohort of patients. Nevertheless, our population is representative of the general DMD population, as the participant characteristics are comparable to literature.

A second limitation relates to our measurements of individual muscle strength. External muscle torque measurements, as we performed with the static frame myometer are unable to measure the maximal torque of isolated muscles. We attempted to mimic the activation of individual muscles as close as possible by choosing measurement positions that primarily required the activation of one muscle, the prime mover. We reported muscles torques as our primary outcome rather than muscle forces, which are more commonly used in literature. Muscle forces, however, do not account for the effect of lever arm, which we believe is more relevant in our study as we measured subjects in a wide age/height range [37]. For comparability we also reported maximal muscle forces in Table 2.

Finally, the use of normalized sEMG amplitudes has some limitations as well. The maximal sEMG amplitude (in MVIC) can be influenced by pain, fear of pain, restrictions in the range of motion and/or motivation [18]. As a result normalized sEMG amplitudes over 100\% MVIC were sometimes seen. This underperformance during MVIC measurements could affect both healthy subject and patients. However, in patients, pain might be of greater influence due to joint contractures. The obtained results, however, show large differences between healthy boys and DMD patients, which cannot be attributed solely to underperformance.

Despite these limitations, we think this study gives valuable and objective insights in UE function and activity level of boys and men with DMD, which are of great clinical importance for the selection and evaluation of suitable interventions.

\section{Conclusions}

The decline of muscle functions precedes the decline in performance of UE activities, and therefore may play a role in early detection of UE limitations. Early detection can have important clinical implications as it allows for starting interventions, such as contracture prevention and physical exercise training, timely and minimize functional decline. Increased sEMG levels demonstrate that DMD patients use more of their muscle capacity compared to healthy subjects to perform daily activities. This might result in increased fatigability, which should receive attention in clinical practice as this is an important determinant of quality of life. Active maximal joint angles are highly related to functional scales, therefore preserving the full range of motion is important in daily life. Monitoring active joint angles can help to select appropriate interventions timely, to minimize UE decline. Finally, the results of this study can be used for the development of new composite outcome measures for clinical trials, that not only aim at the ICF activity level, but also on the ICF level of body functions and structures.

\section{Abbreviations \\ DMD: Duchenne Muscular Dystrophy; ICF: International Classification of Functioning, Disability and Health; MVIC: Maximal voluntary isometric contraction; PUL: Performance of Upper Limb; sEMG: Surface electromyography; UE: Upper extremity}

\section{Acknowledgments \\ The authors would like to thank all participants in this study. We also would like to acknowledge the VU Medical Centre for designing and making the static frame myometer. This research was supported by the Dutch Technology Foundation STW (which is part of the Netherlands Organization for Scientific Research (NWO), and which is partly funded by the Ministry of Economic Affairs), United Parent Projects Muscular Dystrophy, Prinses Beatrix Spierfonds, Spieren voor Spieren, Johanna Kinderfonds, Kinderrevalidatiefonds Adriaanstichting, Focal Meditech, OIM Orthopedie, Ambroise, and Flextension.}

\section{Funding}

This work was supported by the Dutch Technology Foundation STW under grant number 11832.

\section{Availability of data and materials}

Not applicable.

\section{Authors' contributions}

MJ, JH, BK and IdG conceived and designed the experiment. MJ was the main author of the manuscript and JH, BK and IdG contributed to the writing and editing of the manuscript. MJ performed the experiment, analyzed the data and obtained ethical approval. All authors read and approved the manuscript.

\section{Competing interests}

The authors declare that they have no competing interest.

Consent for publication

Not applicable. 


\section{Ethics approval and consent to participate}

This study was approved by the medical ethical committee Arnhem-Nijmegen, the Netherlands (Registration number 2012/135, NL nr.: 39126.091.12). Informed consent was obtained from all participants and from their parents when the subjects were under 18 years of age.

\section{Publisher's Note}

Springer Nature remains neutral with regard to jurisdictional claims in published maps and institutional affiliations.

\section{Author details}

'Department of Rehabilitation, Radboud University Medical Center, Donders Centre for Neuroscience, Reinier Postlaan 4, 6525 GC Nijmegen, The Netherlands. ${ }^{2}$ Department of Rehabilitation Medicine, VU University Medical Center, MOVE Research Institute, Amsterdam, The Netherlands. ${ }^{3}$ Department Biomechanical Engineering, University of Twente, Enschede, The Netherlands.

Received: 5 December 2016 Accepted: 16 May 2017

Published online: 26 May 2017

\section{References}

1. Mendell JR, Lloyd-Puryear M. Report of MDA muscle disease symposium on newborn screening for Duchenne muscular dystrophy. Muscle Nerve. 2013;48:21-6.

2. Bello L, Gordish-Dressman H, Morgenroth LP, Henricson EK, Duong T, Hoffman EP, Cnaan A, McDonald CM, Investigators C. Prednisone/ prednisolone and deflazacort regimens in the CINRG Duchenne Natural History Study. Neurology. 2015;85:1048-55.

3. Mattar FL, Sobreira C. Hand weakness in Duchenne muscular dystrophy and its relation to physical disability. Neuromuscul Disord. 2008;18:193-8.

4. Janssen MM, Bergsma A, Geurts AC, de Groot IJ. Patterns of decline in upper limb function of boys and men with DMD: an international survey. J Neurol. 2014;261:1269-88.

5. Eagle M, Baudouin SV, Chandler C, Giddings DR, Bullock R, Bushby K Survival in Duchenne muscular dystrophy: improvements in life expectancy since 1967 and the impact of home nocturnal ventilation. Neuromuscul Disord. 2002;12:926-9.

6. Eagle M, Bourke J, Bullock R, Gibson M, Mehta J, Giddings D, Straub V, Bushby K. Managing Duchenne muscular dystrophy-the additive effect of spinal surgery and home nocturnal ventilation in improving survival. Neuromuscul Disord. 2007;17:470-5.

7. Balaban B, Matthews DJ, Clayton GH, Carry T. Corticosteroid treatment and functional improvement in Duchenne muscular dystrophy: long-term effect. Am J Phys Med Rehabil. 2005;84:843-50.

8. Connolly AM, Schierbecker J, Renna R, Florence J. High dose weekly oral prednisone improves strength in boys with Duchenne muscular dystrophy. Neuromuscul Disord. 2002:12:917-25.

9. Henricson EK, Abresch RT, Cnaan A, Hu F, Duong T, Arrieta A, Han J, Escolar DM, Florence JM, Clemens PR, et al. The cooperative international neuromuscular research group Duchenne natural history study: glucocorticoid treatment preserves clinically meaningful functional milestones and reduces rate of disease progression as measured by manual muscle testing and other commonly used clinical trial outcome measures. Muscle Nerve. 2013:48:55-67.

10. Pane M, Fanelli L, Mazzone ES, Olivieri G, D'Amico A, Messina S, Scutifero M, Battini R, Petillo R, Frosini S, et al. Benefits of glucocorticoids in non-ambulant boys/men with Duchenne muscular dystrophy: A multicentric longitudinal study using the Performance of Upper Limb test. Neuromuscul Disord. 2015;25:749-53.

11. Jansen M, van Alfen N, Geurts AC, de Groot IJ. Assisted bicycle training delays functional deterioration in boys with Duchenne muscular dystrophy: the randomized controlled trial "no use is disuse". Neurorehabil Neural Repair. 2013;27:816-27.

12. Alemdaroglu I, Karaduman A, Yilmaz OT, Topaloglu H. Different types of upper extremity exercise training in Duchenne muscular dystrophy: effects on functional performance, strength, endurance, and ambulation. Muscle Nerve. 2015:51:697-705.

13. Jansen M, Burgers J, Jannink M, Alfen N, Groot IJM: Upper Limb Training with Dynamic Arm Support in Boys with Duchenne Muscular Dystrophy: A Feasibility Study. Int J Phys Med Rehabil. 2015;3:256.
14. World Health O: ICF : International classification of functioning, disability and health / World Health Organization. Geneva: World Health Organization; 2001.

15. Brooke MH, Griggs RC, Mendell JR, Fenichel GM, Shumate JB, Pellegrino RJ. Clinical trial in Duchenne dystrophy. I. The design of the protocol. Muscle Nerve. 1981:4:186-97.

16. Mayhew A, Mazzone ES, Eagle M, Duong T, Ash M, Decostre V, Vandenhauwe M, Klingels K, Florence J, Main M, et al. Development of the Performance of the Upper Limb module for Duchenne muscular dystrophy. Dev Med Child Neurol. 2013;55:1038-45.

17. Hermens HJ. Freriks B, sselhorst-Klug C, Rau G: Development of recommendations for SEMG sensors and sensor placement procedures. J Electromyogr Kinesiol. 2000;10:361-74.

18. Cram JR, Criswell E: Cram's introduction to Surface Electromyography. 2nd ed. Burlington: Jones and Bartlett Publishers; 2011.

19. De Luca CJ, Gilmore LD, Kuznetsov M, Roy SH. Filtering the surface EMG signal: Movement artifact and baseline noise contamination. J Biomech. 2010;43:1573-9.

20. Winter DA, Yack HJ. EMG profiles during normal human walking: stride-to-stride and inter-subject variability. Electroencephalogr Clin Neurophysiol. 1987;67:402-11.

21. Jansen M, van Alfen N. Nijhuis van der Sanden MW, van Dijk JP, Pillen S, de Groot IJ: Quantitative muscle ultrasound is a promising longitudinal follow-up tool in Duchenne muscular dystrophy. Neuromuscul Disord. 2012;22:306-17.

22. Duffy M, Munro B, Jacobsen B. Key principles of statistical inference. In: Munro BLEA, editor. Statistical methods for health care research. 5th ed. Philadelphia: Lippincott Williams \& Wilkins; 2005. p. 73-107.

23. Scholten RR, Pillen S, Verrips A, Zwarts MJ. Quantitative ultrasonography of skeletal muscles in children: normal values. Muscle Nerve. 2003;27:693-8.

24. Jaspers E, Feys H, Bruyninckx H, Harlaar J, Molenaers G, Desloovere K. Upper limb kinematics: development and reliability of a clinical protocol for children. Gait Posture. 2011;33:279-85.

25. James W, Orr JF. Upper limb weakness in children with Duchenne muscular dystrophy-a neglected problem. Prosthet Orthot Int. 1984;8:111-3.

26. Lord JP, Portwood MM, Lieberman JS, Fowler Jr WM, Berck P. Upper extremity functional rating for patients with Duchenne muscular dystrophy. Arch Phys Med Rehabil. 1987:68:151-4.

27. Bartels B, Pangalila RF, Bergen MP, Cobben NA, Stam HJ, Roebroeck ME. Upper limb function in adults with Duchenne muscular dystrophy. J Rehabil Med. 2011;43:770-5.

28. Zaidman CM, Connolly AM, Malkus EC, Florence JM, Pestronk A. Quantitative ultrasound using backscatter analysis in Duchenne and Becker muscular dystrophy. Neuromuscul Disord. 2010;20:805-9.

29. Rutkove SB, Geisbush TR, Mijailovic A, Shklyar I, Pasternak A, Visyak N, Wu JS, Zaidman C, Darras BT. Cross-sectional evaluation of electrical impedance myography and quantitative ultrasound for the assessment of Duchenne muscular dystrophy in a clinical trial setting. Pediatr Neurol. 2014;51:88-92.

30. McDonald CM, Abresch RT, Carter GT, Fowler Jr WM, Johnson ER, Kilmer DD, Sigford BJ. Profiles of neuromuscular diseases. Duchenne muscular dystrophy. Am J Phys Med Rehabil. 1995:74:S70-92.

31. Skalsky AJ, McDonald CM. Prevention and management of limb contractures in neuromuscular diseases. Phys Med Rehabil Clin N Am. 2012;23:675-87.

32. Wei Y, Speechley KN, Zou G, Campbell C: Factors Associated With HealthRelated Quality of Life in Children With Duchenne Muscular Dystrophy. J Child Neurol. 2016:31:879-86.

33. Nordlund MM, Thorstensson A, Cresswell AG. Central and peripheral contributions to fatigue in relation to level of activation during repeated maximal voluntary isometric plantar flexions. J Appl Physiol (1985). 2004;96:218-25.

34. Aizawa J, Masuda T, Koyama T, Nakamaru K, Isozaki K, Okawa A, Morita S. Three-dimensional motion of the upper extremity joints during various activities of daily living. J Biomech. 2010:43:2915-22.

35. Romilly D, Anglin C, Gosine R, Hershler C, Raschke S. A Functional Task Analysis and Motion Simulation for the Development of a Powered Upper-Limb Orthosis. IEEE trans rehabil eng. 1994;2:119-29.

36. van Andel CJ, Wolterbeek N, Doorenbosch CA, Veeger DH, Harlaar J. Complete 3D kinematics of upper extremity functional tasks. Gait Posture. 2008;27:120-7

37. Hebert LJ, Maltais DB, Lepage C, Saulnier J, Crete M. Hand-Held Dynamometry Isometric Torque Reference Values for Children and Adolescents. Pediatr Phys Ther. 2015:27:414-23. 